\title{
Dietary interventions as a neuroprotective therapy for the delay of the onset of cognitive decline in older adults: Evaluation of the evidence
}

\author{
Debra Krause* and Peter Roupas
}

CSIRO Agriculture and Food, Private Bag 16, 671 Sneydes Road, Werribee 3030, Victoria Australia

Corresponding Author: Debra Krause, CSIRO Agriculture and Food, Werribee 3030, Victoria, Australia

Submission Date: June $14^{\text {th }}$, 2017, Acceptance Date: October $27^{\text {th }}$, 2017, Publication Date: October $31^{\text {st }}, 2017$

Citation: Krause D., Roupas P., Dietary interventions as a neuroprotective therapy for the delay of the onset of cognitive decline in older adults: Evaluation of the evidence. Functional Foods in Health and Disease 2017; 7(10):743-757. https://doi.org/10.31989/ffhd.v7i10.370

\begin{abstract}
Background: The relationship between nutrition and cognitive functioning is unclear, especially in elderly populations as many elderly people with cognitive impairment have low blood levels of some nutrients even in the absence of malnourishment. The objective of this review was to assess the evidence from systematic reviews of human studies on the effectiveness of dietary interventions as monotherapies in delaying the onset of cognitive decline in older adults.
\end{abstract}

Scope and approach: Evidence-based methodologies were used to gather and assess the highest levels of evidence that evaluated the effects of administration of any dose of the individual dietary interventions as neuroprotective agents for any duration. The search strategy was designed to identify systematic reviews and meta-analyses published from 1990 to December 2015. There were no language restrictions as part of the inclusion criteria.

Key Findings and Conclusions: This review provides the current state of knowledge from systematic reviews on the effects on cognition of acetyl-L-carnitine, alpha-lipoic acid, choline, inositol compounds, omega-3 polyunsaturated fatty acids, and polyphenols, which are all commonly studied nutrients for neurocognitive effects. A critical evaluation of the current evidence from systematic reviews indicated that there are no clinically-relevant effects from supplementation with these nutrients on delaying the onset of cognitive decline in older adults.

Keywords: diet, cognition, Alzheimer's disease, dementia, nutrient 


\section{INTRODUCTION}

Nutrition is one of the lifestyle factors that may contribute to the development and progression of chronic diseases such as atherosclerosis, cancer, cardiovascular disease, diabetes, and neurodegenerative diseases [1]. Increasing life expectancy has also correlated with an increasing number of people affected by chronic diseases associated with aging, such as dementia. Neurodegenerative diseases such as Alzheimer's Disease (AD) are reported to be responsible for up to $80 \%$ of dementia cases. Age is the major risk factor for AD, with the prevalence doubling every 5 years after the age of 65 [2].

Possible mechanisms associated with neurodegeneration have been hypothesised with the view of determining the cause of abnormalities in the transport, degradation, and aggregation of proteins including amyloid- $\beta$ peptides, apolipoprotein $\mathrm{E}$ (APOE) and tau, a microtubuleassociated protein in the brain. Free radicals and neuroinflammatory processes have been hypothesised as a cause of many of the neurodegenerative conditions [3]. Natural compounds including acetyl-L-carnitine, carotenoids, curcumin, vitamin D and polyphenols, and other dietary components have been studied for their potential to target AD pathways [1]. Many of these natural compounds are widely available as nutritional supplements and have been studied for their potential role in neuroprotection. However, the relationship between nutrition and cognitive functioning, especially in elderly populations, is unclear as many elderly people with cognitive impairment have low blood levels of some nutrients even in the absence of malnourishment $[4,5]$. These findings have been reported in cross-sectional and longitudinal studies and then further assessed in systematic reviews and meta-analyses to pool results to achieve some definitive conclusions.

The objective of this review therefore was to evaluate the highest levels of evidence, namely systematic reviews of human studies, of the effectiveness of dietary interventions as monotherapies in delaying the onset of cognitive decline in older adults. The key dietary components identified for inclusion in this review commonly associated for their potential effects on prevention of cognitive decline were acetyl-L-carnitine, alpha-lipoic acid, choline, inositol compounds, omega-3 polyunsaturated fatty acids (omega-3 PUFA), and polyphenols. These dietary components were chosen as there were a significant number of studies available in relation to cognitive function. The umbrella review approach was used, as it enabled the overall assessment of a broad scope of nutrients that have been associated with a delay in the onset of cognitive decline to be critically evaluated and summarized in a single review.

\section{METHODS}

\section{Search Strategy}

Literature searches were undertaken on alpha-lipoic acid, acetyl-L-carnitine, choline, inositol compounds, omega-3 PUFA, and polyphenols and their effect on cognitive decline using the process previously described [5,6]. Databases searched were; Medline on PubMed, Web of Science, Cochrane Central (Database of Systematic Reviews and Cochrane Collaboration Central Register of Controlled Trials), Centre for Reviews and Dissemination Databases (Database of Reviews of Effects (DARE)), National Health Service Economic Evaluation Database (NHS EED) and Health Technology Assessment Database), and Joanna Briggs Institute (JBI) Library of Systematic Reviews and the Agency for Healthcare Research and Quality (US Department of 
Health and Human Services). Searches spanned_1990 to December 2015 without any language restriction, with 1990 being selected as a conservative estimate for the earliest likely publication of a systematic review on this topic, as this pre-dates some of the earliest work in this field.

\section{Inclusion and exclusion criteria Population}

This review considered systematic reviews of adult humans, who were mean age 50 years or older without clinically-diagnosed dementia/AD at baseline, including memory complainers and/or with mild cognitive impairment (MCI) as a result of the aging process, but not with cognitive impairment due to brain injury. Studies of older adults with AD /dementia were discussed, where there were no systematic reviews identified for adults without dementia/AD at baseline. Studies of participants with vascular dementia or studies using animal models were not considered for this review.

\section{Types of interventions / phenomena of interest}

This review considered systematic reviews that evaluated the effects of the intake of any dose of the following individual dietary components as neuroprotective agents for any duration. Included in the review were alpha-lipoic acid, acetyl-L-carnitine, choline, inositol compounds, omega-3 PUFA, and polyphenols. The review did not consider systematic reviews of combinations of dietary components or supplements, vitamin supplements, studies that combined drugs with a dietary intervention or studies of whole-of-diet approaches.

\section{Types of outcomes}

The review considered studies that included primary outcome measures from a range of cognitive function tests, brain imaging techniques, blood biomarkers of MCI and AD risk as previously described $[5,6]$

\section{Types of studies}

The review considered published systematic reviews and meta-analyses on the listed dietary components.

\section{Data extraction and synthesis}

Studies identified for retrieval were assessed by two independent reviewers for methodological quality as per JBI methodology [7] prior to inclusion in the review. Data extracted from papers included details of the populations, interventions, study methods, and outcomes of significance to the question of this review article and specific objectives. The data were extracted and listed by intervention and levels of evidence using the National Health and Medical Research Council (NHMRC, Australia) criteria [8] as previously described [5]. While studies were categorized by study design, it should be emphasized that this does not automatically translate to the strength of the evidence, due to other contributing factors that were also assessed. Meta-analyses was not able to be conducted due to heterogeneity of the study designs, confounding factors that were not controlled for and the subjective measure of some end-point determinations.

\section{RESULTS AND DISCUSSION}


A summary of the studies evaluating the effects of acetyl-L-carnitine, alpha lipoic acid, choline, inositol compounds, and polyphenols on prevention of cognitive decline is shown in Error! Reference source not found. 1. 
Table 1. Studies of dietary interventions as monotherapies on cognitive decline

\begin{tabular}{|c|c|c|c|}
\hline Study & Study type & $\begin{array}{l}\text { Level of } \\
\text { Evidence }^{1}\end{array}$ & Outcome \\
\hline \multicolumn{4}{|l|}{ Acetyl -L-carnitine } \\
\hline Hudson \& Tabet 2003 (updated 2007) [10] & $\begin{array}{l}\text { Systematic review } \\
\text { (Cochrane) }\end{array}$ & I & $\begin{array}{l}\text { Sixteen RCTs of 30-431 participants, 40-97 years of age and 3-12 months duration, found } \\
\text { no evidence of benefit of ALC in the areas of cognition, severity of dementia, functional } \\
\text { ability or Clinical Global Impression as a continuous measure. }\end{array}$ \\
\hline Montgomery et al 2003[11] & $\begin{array}{l}\text { Systematic review } \\
\text { with meta-analysis }\end{array}$ & I & $\begin{array}{l}\text { Meta-analysis of } 21 \text { RCTs of } 12-183 \text { participants, } 71.9 \pm 6.5 \text { years of age and 3-12months } \\
\text { duration found a significant beneficial effect of ALC compared to placebo on both clinical } \\
\text { scales and psychometric tests in MCI and/or mild AD. }\end{array}$ \\
\hline \multicolumn{4}{|l|}{ Alpha lipoic acid } \\
\hline Klugman et al 2004 (updated 2007) [13] & $\begin{array}{l}\text { Systematic review } \\
\text { (Cochrane) }\end{array}$ & I & $\begin{array}{l}\text { There were no RCTs identified investigating ALA for dementia. No current evidence of } \\
\text { efficacy of ALA for dementia. }\end{array}$ \\
\hline \multicolumn{4}{|l|}{ Choline } \\
\hline Higgins and Flicker 2000 (updated 2007) [15] & $\begin{array}{l}\text { Systematic review } \\
\text { (Cochrane) }\end{array}$ & I & $\begin{array}{l}\text { Ten RCTs of 10-63 participants, } 47-89 \text { years of age and } 1 \text { week }-2 \text { years duration, found no } \\
\text { clear clinical benefit of lecithin for AD. A further RCT of } 90 \text { participants with subjective } \\
\text { memory problems, mean age } 55 \text { years and } 8 \text { weeks duration showed a significant positive } \\
\text { effect of lecithin on memory but requires verification through further trials. }\end{array}$ \\
\hline \multicolumn{4}{|r|}{, } \\
\hline \multicolumn{4}{|l|}{ No systematic reviews published } \\
\hline \multicolumn{4}{|l|}{ Polyphenols } \\
\hline Lamport et al 2012[25] & Systematic review & III- $2 / 3^{2}$ & $\begin{array}{l}\text { Fifteen RCTs of 9-175 participants, } 47-80+\text { years of age and } 6 \text { weeks }-12 \text { months duration } \\
\text { and } 4 \text { cohort studies of } 882-2,574 \text { elderly participants, duration } 3 \text { months }-13 \text { years } \\
\text { evaluated the association of consumption of a range of polyphenols on cognitive function } \\
\text { on healthy or MCI adults. No clear evidence for an association between cognitive outcomes } \\
\text { and polyphenol dose response, duration of intervention, or population studied. }\end{array}$ \\
\hline Rafnsson et al 2013[24] & Systematic review & III-3 & $\begin{array}{l}\text { Two dietary intake studies of } 342 \text { and } 1640 \text { elderly participants for } 3 \text { and } 10 \text { years assessing } \\
\text { the effects of flavonoids on cognitive decline, found a lower rate of cognitive decline with } \\
\text { increased intake and no association respectively. }\end{array}$ \\
\hline Scholey and Owen 2013 [26] & Systematic review & II & $\begin{array}{l}\text { One RCT of } 101 \text { participants, } \geq 60 \text { years of age and } 6 \text { weeks duration found no difference in } \\
\text { cognitive performance following consumption of a chocolate bar ( } 397 \mathrm{mg} \text { proanthocyanins) } \\
\text { and cocoa drink ( } 357 \mathrm{mg} \text { proanthocyanins) compared to placebo. }\end{array}$ \\
\hline
\end{tabular}

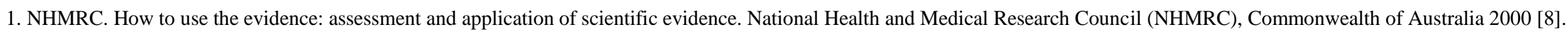

2. This systematic review evaluated studies representing different levels of evidence

Abbreviations: ALC,Acetyl -L-carnitine; RCT, randomised controlled trial; MCI, mild cognitive impairment; AD, Alzheimer's disease; ALA, Alpha lipoic acid 


\section{Acetyl-L-carnitine}

Acetyl-L-carnitine (ALC) is an acetylated form of L-carnitine that is naturally produced by the body; however, it is also taken as a dietary supplement. The effects of ALC on cognitive decline have been investigated in clinical trials, following studies that reported ALC offering protective effects to peripheral and nervous system synapses in neurodegenerative and aging animal models [9].

A Cochrane review conducted in 2003 and last assessed in November 2007, with the most recent report stating that there was some evidence for the benefit of ALC on Clinical Global Impression (CGI) at 12 and 24 weeks but not at 52 weeks, and a statistically significant treatment effect on Mini-Mental State Examination (MMSE) at 24 weeks but not at 12 or 52 weeks. Metaanalyses of the most commonly observed adverse effects (gastrointestinal effects) found no statistically significant differences between treated and control groups. The authors concluded that there was no evidence of benefit of ALC on cognition, severity of dementia, functional ability, or CGI as a continuous measure [10].

A meta-analysis of 21 double-blind, placebo-controlled prospective, parallel group comparison studies of at least 3 months duration with daily doses of $1.5-3.0 \mathrm{~g} /$ day in MCI and/or mild AD patients reported a significant beneficial effect of ALC compared to placebo on both clinical scales and psychometric tests over a six month period [11]. Meta analyses were conducted on clinical and psychometric tests individually in addition to a combined effect. The effect size (ES) for clinical global impression of change was significant, [ES $=0.32,95 \%$ confidence interval $(C I)=0.18-0.47]$ while the ES for psychometric tests only became significant when drawing and learning tests were excluded from the analysis. When clinical and psychometric tests were combined into an integrated summary effect, a significant effect size of [ES $=0.201,95 \%$ confidence interval $(\mathrm{CI})=0.107-0.295]$ was observed. The effects were seen at the time of first assessment ( 3 months) and increased at the six month assessment of 16 studies; ALC was also well tolerated in all studies [11].

These two systematic reviews assessed fourteen of the same studies; however, Hudson et al [10] included studies of any type of dementia and severity, while Montgomery et al [11] included only studies of MCI and mild AD as a diagnosis, due to previous studies reporting that ALC may be more effective in milder AD cases. The two reviews reached similar conclusions at 6 months. The importance of bioavailability studies to inform dose selection was also raised, due to the possible breakdown of ALC in both the gastrointestinal tract and liver having varying effects on the plasma levels of ALC in different individuals. Hudson and Tabet [10] found significant difference between the ALC and control groups at 12 months, but this assessment was not reported by Montgomery et al [11]. This raised the query of the sustained effect of ALC on cognitive outcomes, although it may also be due to ALC being more effective in milder AD cases. Therefore, the current evidence suggests the need for further studies to clarify the benefit on cognition in older adults from taking this supplement.

\section{Alpha-lipoic acid}

Alpha lipoic acid (ALA) has been postulated as a potential therapeutic agent in AD due to its ability to increase acetylcholine production by activation of choline acetyltransferase, chelate redox-active transition metals, and scavenge reactive oxygen species [12]. 
A Cochrane review has assessed the role and clinical efficacy of ALA in the treatment of dementia. The review was undertaken in 2004 and last assessed up to date in 2008. As there were no randomized double-blind placebo-controlled trials (RCTs) investigating ALA for dementia, the authors concluded that there was no evidence of efficacy of ALA for dementia, and that therefore ALA could not be recommended for the treatment of any type of dementia [13]. Since this Cochrane review, there has been no additional evidence established. Therefore, the conclusion remains valid, with there currently being no evidence for the role of ALA in the prevention of cognitive decline in older adults.

\section{Choline}

Previous studies have determined that those who suffer from AD lack the enzyme responsible for converting choline into acetylcholine within the brain, and have hypothesized that lecithin, the major dietary source of choline, may fast-track the synthesis of acetylcholine in the brain through increased availability of choline [14].

The effectiveness of lecithin for the treatment of dementia or cognitive impairment was evaluated in a Cochrane review [15]. Although out of the scope of this review, an assessment of ten randomized trials involving 265 participants with AD found no significant benefit on memory performance. However, a further single study of 90 participants with subjective memory problems reported a significantly positive result for lecithin [15]. The authors concluded that the evidence from randomized trials did not support the use of lecithin in the treatment of patients with dementia. Further trials are required to evaluate the role of lecithin in patients with subjective memory complaints to determine any clinical benefit of lecithin in the delay of cognitive decline. No additional evidence has been published since this Cochrane review.

\section{Inositol}

Scyllo-inositol has been found to inhibit cognitive deficits in mice and to significantly decrease insoluble amyloid $-\beta 40$ peptide, amyloid $-\beta 42$ peptide, and plaque accumulation in mice brains [16]. Previously, clinical trials have been undertaken using a range of doses of inositol or scyolloinositol [17]. However, there were no systematic reviews identified related to inositol and its effectiveness in delaying the onset of cognitive decline in older adults.

\section{Polyphenols}

Polyphenols are a group of naturally occurring secondary metabolites of plants, and are characterized by the presence of one or more phenolic units per molecule. Several classes of polyphenols have been assessed for their potential anti-aging and neuroprotective properties. These include flavonoids such as the anthocyanins that are commonly found in berries, grapes, and red wine, in addition to catechins which are typically found in tea [18]. Examples of bioactives from the non-flavonoids class of bioactives includes curcumin from turmeric and resveratrol from grapes and red wine. Curcumin has been reported to have a range of effects including, decreasing beta-amyloid plaques, delaying degradation of neurons, metal-chelation, anti-inflammatory, antioxidant and decreasing microglia formation, in addition to the improvement of the overall memory in patients with AD [19]. From in vitro studies grape seed polyphenolic extracts have been shown to offer beneficial disease-modifying bioactivities in tau-associated neurodegenerative 
disorders [20]. Resveratrol has been shown to have effects in in vitro models of AD and in in vivo animal studies, while moderate red wine consumption has been associated with a lower incidence of dementia and AD in epidemiological studies [21], though clinical studies in humans are lacking. Through epidemiological studies, it has been reported that daily consumption of up to three servings of wine is associated with a lower AD risk in the elderly without the APOE $\varepsilon 4$ allele [22] and moderate drinkers with MCI consuming less than 1 serving of wine per day demonstrated a significantly lower rate of progression to dementia compared to non-drinkers [23].

A systematic review of population-based cohort studies examined the association of antioxidant nutrients with cognitive decline in older people [24]. Two of the studies included in the systematic review used dietary intake information to determine if there was any relationship between flavonoids and cognitive decline. While a three-year study found no association between a higher dietary level of flavonoids and a reduced likelihood of decline in global cognitive function, a second study observed that for participants with high (3rd and 4th quartiles) total dietary flavonoid intake there was a slower rate of global cognitive decline over 10 years.

Although out of the scope of this review, a systematic review [25] evaluated the effect of consumption of a range of polyphenols on cognitive function on healthy or mildly cognitively impaired adults. Studies on adults 50 years and over included 3 berry juice studies, 1 cocoa study, 10 isoflavone supplement studies, 1 flavonoid supplement study, and 4 epidemiological studies. While two of the three berry fruit juice studies showed potential cognitive benefits particularly for immediate verbal memory, the studies enrolled only up to 12 participants and lasted only 12weeks. For cocoa there was no evidence from a 6-week study that suggested cocoa polyphenols were associated with cognitive benefits. While this outcome was also supported in a further systematic review [26], the authors suggested that the lack of positive findings may be attributed to study methodology. Limitations in study methodology included variations in the types of food frequency questionnaires and foods used to estimate polyphenol intake, in addition to differences in the methods used to calculate polyphenol intake. While study methodology may have been a contributing factor to the findings, it is also important to note that 12 -weeks is not sufficient duration to study cognitive decline as there is not likely to be any measurable cognitive decline in participants over such a short time frame; therefore this is a significant limitation of this study and other studies with short durations.

Soy protein, soy isoflavone supplements, and /or soy milk, were used for all isoflavone based interventions, except for one intervention that utilized a red clover supplement [25]. While studies showed significant improvements in the cognitive domains of delayed spatial memory (4 studies) and executive function (4 studies), lack of consistency in isoflavone dose, trial duration, cognitive tests used, and cognitive testing procedures, led to inconclusive findings for the cognitive benefit of isoflavones. A trial of 101 adults, mean age 68 years using a flavonoid supplement, an extract of French maritime pine bark (Pycnogenol®) found benefits in spatial working memory compared to a placebo after 3 months.

Three of the four epidemiological studies assessed by Lamport et al [25], which were between 1 and 13 years in duration, observed both positive and negative associations with cognitive function, while a 3 month study found no association between flavonoid intake and the cognitive domains of memory, fluid intelligence, or verbal fluency. Comparison between studies was difficult due to variations in the types of food frequency questionnaires used, the differences in the 
methods and databases used to calculate polyphenol intake, and the lack of detail reported for cognitive testing procedures, which is a significant limitation in evaluating the evidence for polyphenol intake.

Following evaluation of the range of polyphenol sources, Lamport et al [25] concluded that declarative memory, particularly spatial memory, appears to be the most sensitive to polyphenol consumption and the effects may vary depending on polyphenol source. Overall, 11 studies reported cognitive benefits in some cognitive domains following polyphenol consumption.

While the findings indicate that polyphenol consumption may have some potential to benefit cognition, comparison between studies has not been possible due to methodological inconsistencies. Accordingly, no clear evidence could be established for an association between cognitive outcomes and polyphenol dose response, length of intervention, or populations studied.

\section{Omega-3 PUFA}

Omega-3 polyunsaturated acids are essential fatty acids, and docosahexanoic acid (DHA) is a major component of the membrane phospholipids in the brain, and has been widely reported to have anti-atherogenic, anti-inflammatory, anti-amyloid, and neuroprotective properties [27]. Through the evidence assessed from an array of observational and epidemiological studies, a possible association between high dietary intake of PUFA and lower risk of dementia has been proposed [28-30]. A summary of the studies evaluating the effects of omega-3 PUFA on cognitive outcomes for the prevention of cognitive decline is shown in Table 2.

Four systematic reviews / meta-analyses representing level one evidence were identified evaluating the effectiveness of omega-3 PUFA in delaying the onset of cognitive decline in older adults.

A Cochrane review published in 2012 evaluated the effect of omega-3 PUFA supplementation for the prevention of dementia and cognitive decline in cognitively healthy people over the age of 60 years at study commencement [31]. This updates a previous Cochrane review completed in 2006 [32]. The Cochrane review included a total of 3536 participants from three randomized controlled trials of studies between 6 and 40 months in duration. Two studies delivered omega-3 PUFA in capsule form to participant diets, while the third study used a margarine spread. Placebos were either olive oil or sunflower oil capsules or margarine. The main outcomes of interest were cognitive decline, new dementia cases diagnosed over the course of the study, side effects, and adherence to the intervention.

Analysis of two studies involving 3221 participants found no difference between the omega3 PUFA and placebo group in MMSE score at final follow-up, [mean difference -0.07. (95\%CI 0.25-0.10)]. Similarly, other tests of cognitive function such as word learning and verbal fluency showed no beneficial effect of omega-3 PUFA supplementation in three studies of 1043 participants. Furthermore, it was observed that participants in both the intervention and control groups experienced little or no cognitive decline over the duration of the study. A possible reason for this relates to the use of olive oil as a placebo for those studies. Olive oil has confirmed heart health benefits which have been reported to be of possible benefits to brain health (and cognition) [33]. 
Table 2. Studies of dietary interventions as monotherapies, on cognitive decline - omega -3 polyunsaturated fatty acids

\begin{tabular}{|c|c|c|c|}
\hline Study & Study type & $\begin{array}{l}\text { Level of } \\
\text { Evidence }^{1}\end{array}$ & Outcome \\
\hline \multicolumn{4}{|l|}{ Omega-3 PUFA } \\
\hline $\begin{array}{l}\text { Sydenham et al 2012[31] } \\
\text { (update of Lim et al 2006) } \\
\text { [32] }\end{array}$ & $\begin{array}{l}\text { Systematic review } \\
\text { (Cochrane) }\end{array}$ & I & $\begin{array}{l}\text { Three RCTs of 299-2,493 participants, } 60+\text { years and 6-40 months duration, found no evidence of benefit of omega-3 PUFA } \\
\text { supplementation on cognitive function among cognitively healthy participants over the age of } 60 \text { years. }\end{array}$ \\
\hline Jiao et al 2014[35] & $\begin{array}{l}\text { Systematic review } \\
\text { with meta- analysis }\end{array}$ & I & $\begin{array}{l}\text { Meta-analysis of } 12 \text { RCTs of 52-581 participants, } 68.9-74.2 \text { years and } 5.9-19.5 \text { months duration, found no evidence of an effect of } \\
\text { omega-3 PUFA supplementation on significantly improving cognitive performance. There was a significant difference in the } \\
\text { specific cognitive domain of attention with omega-3 PUFA supplementation. }\end{array}$ \\
\hline Mazereeuw et al 2012[34] & $\begin{array}{l}\text { Systematic review } \\
\text { with meta- analysis }\end{array}$ & I & $\begin{array}{l}\text { Meta-analysis of } 10 \text { RCTs of } 21-495 \text { participants, }>50 \text { years of age and 15-108 weeks duration, found evidence of an effect of } \\
\text { omega-3 PUFA supplementation within specific cognitive domains in MCI, but not in healthy or AD subjects. }\end{array}$ \\
\hline Yurko-Mauro et al 2015[36] & $\begin{array}{l}\text { Systematic review } \\
\text { with meta- analysis }\end{array}$ & I & $\begin{array}{l}\text { Meta-analysis of } 11 \text { RCTs of } 8-867 \text { participants, mean age }>50 \text { and } 42-730 \text { days, found that DHA/EPA supplementation } \\
\text { significantly improved memory outcomes of adults with mild memory complaints }(\mathrm{P}<0.004) .1 \mathrm{~g} / \text { day DHA/EPA improved } \\
\text { episodic memory }(\mathrm{P}<0.04) \text {, regardless of cognitive status at baseline. DHA was associated with semantic and working memory } \\
\text { changes from baseline. }\end{array}$ \\
\hline $\begin{array}{l}\text { de Souza Fernandes et } \\
\text { al } 2015 \text { [41] }\end{array}$ & Systematic review & II & $\begin{array}{l}\text { Four RCTs of 20-295 participants } 72.6-85 \text { years, and 12-18months duration found evidence of an effect of omega-3 PUFA } \\
\text { supplementation for verbal fluency and attention domains in MCI, and for non-APOE\&4 genotype but not in AD subjects. }\end{array}$ \\
\hline Wu et al [37] & $\begin{array}{l}\text { Systematic review } \\
\text { with meta- analysis }\end{array}$ & III-3 & $\begin{array}{l}\text { Meta-analysis of } 5 \text { prospective cohort studies of } 488-8,085 \text { participants, } \geq 55 \text { years of age and } 2.1-9.6 \text { years duration, found no } \\
\text { significant association between omega-3 PUFA intake and AD risk. A higher intake of fish was associated with a lower risk of } \\
\text { AD. }\end{array}$ \\
\hline Lin et al 2012[43] & $\begin{array}{l}\text { Systematic review } \\
\text { with meta- analysis }\end{array}$ & III-3/IV 2 & $\begin{array}{l}\text { A meta-analysis of } 10 \text { observational studies of } 10-725 \text { elderly participants found that the levels of EPA, DHA and total omega- } 3 \\
\text { PUFA were lower in patients with dementia. A further meta-analysis of } 5 \text { studies found that the levels of EPA, were significantly } \\
\text { lower in patients with predementia syndrome. }\end{array}$ \\
\hline Dangour et al 2010[42] & Systematic review & II, III- $3^{2}$ & $\begin{array}{l}\text { Eight prospective cohort studies of 79-5,395 participants }>50 \text { years of 3.9-21 years duration/follow up and } 4 \text { RCTs of 20-174 } \\
\text { participants, }>50 y e a r s \text { and 1-12months duration found insufficient evidence for an association of omega-3 PUFA with cognitive } \\
\text { decline or dementia. }\end{array}$ \\
\hline Williams et al 2010[2] & Systematic review & III-3 & $\begin{array}{l}\text { Two systematic reviews and two cohort studies } 404-7,814 \text { participants of } 3-6 \text { years duration suggest a possible association } \\
\text { between higher consumption of omega-3 PUFA and less cognitive decline. }\end{array}$ \\
\hline Fotuhi et al 2009 [38] & Systematic review & III-3 & $\begin{array}{l}\text { Three cohort studies of } 210-3,718 \text { elderly participants of } 4-6 \text { years duration reported that omega-3 PUFA was associated with a } \\
\text { modest benefit in slowing cognitive decline in elderly individuals without dementia. }\end{array}$ \\
\hline $\begin{array}{l}\text { Issa et al 2006[39] } \\
\text { Maclean et al 2005[40] }\end{array}$ & Systematic review & III-3 & $\begin{array}{l}\text { One RCT of } 20 \text { elderly males of } 12 \text { months duration and } 4 \text { cohort studies of } 815-5,386 \text { elderly participants of } 12-46.8 \text { months } \\
\text { duration assessing the effects of omega-3 PUFA intake found insufficient evidence to determine the effects of omega-3 PUFA } \\
\text { on cognitive function in normal ageing or on the incidence or treatment of dementia, although there was limited evidence } \\
\text { suggesting a possible association between omega-3 PUFA and reduced risk of dementia. }\end{array}$ \\
\hline Yurko-Mauro et al 2015[36] & Systematic review & III-3/IV ${ }^{2}$ & $\begin{array}{l}\text { Ten observational studies of } 79-2251 \text { elderly participants, mean age }>50 \text { years, of } 3 \text { months to } 50 \text { years duration, supported } \\
\text { an association between intake/blood levels of DHA/EPA and memory function. }\end{array}$ \\
\hline
\end{tabular}

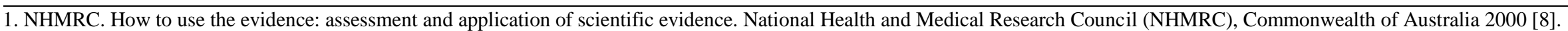

2. This systematic review evaluated studies representing different levels of evidence

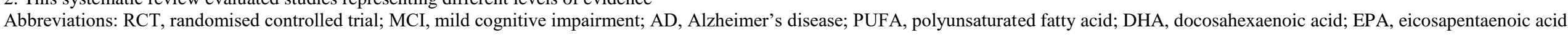


A meta-analysis of the neuropsychological benefit of omega-3 PUFA supplementation in ten RCTs of healthy, mild cognitive impairment or $\mathrm{AD}$ participants of at least 50 years of age was undertaken by Mazereeuw et al [34]. The study quantitatively assessed specific neuropsychological domains, and Hedge's g was used to provide a standardized estimate of effect size for combining the multiple cognitive tests. The results showed that there was no effect of omega-3 PUFA on composite memory ( $\mathrm{g}=0.04$ [95\% CI: $-0.06-0.14], \mathrm{N}=934 / 812, \mathrm{p}=0.452$ ). When examined by domain, no overall benefit was observed for immediate recall, delayed recall, recognition, working memory and executive function, or attention and processing speed. Similarly, no benefits were observed in measures of global cognitive function using MMSE or Alzheimer's Disease Assessment Scale-Cognitive Subscale (ADAS-cog). However, sub-group analysis revealed that when assessed by diagnosis, a benefit of omega-3 PUFA supplementation was detected in MCI subjects for immediate recall (0.16 [0.01-0.32], $\mathrm{N}=349 / 327, \mathrm{p}=0.034)$ and for attention and processing speed $(0.32$ [0.032-0.61], $\mathrm{N}=107 / 86, \mathrm{p}=0.035)$, but not for healthy subjects. There were no benefits observed for delayed recall, recognition memory, or working memory and executive function. Treatment did not benefit $\mathrm{AD}$ patients as measured by the MMSE or ADAS-cog [34].

Although out of the scope of this review, a further meta-analysis [35] assessing many of the same studies (eight studies overlapped), reached similar conclusions of no evidence of an effect of omega-3 PUFA supplementation on significantly improving cognitive performance. Similarly, the meta-analysis also showed a significant difference in the specific cognitive domain of attention with omega-3 PUFA supplementation (Standardised mean difference: 0.13; [95\% CI: 0.01-0.25]). However, this meta-analysis included both cognitively healthy and MCI participants, which differed from that of Mazereeuw et al that included only MCI participants [34].

A meta-analysis of five studies [36] found that DHA supplementation, either alone or in combination with eicosapentaenoic acid (EPA), significantly improved episodic memory of adults $>45$ years with MCI. This supports the study previously reported by Mazereeuw et al [34], where immediate recall in the sub-category of episodic memory, was significantly improved following supplementation for participants with MCI at baseline. Omega-3 PUFA was significantly associated with semantic and working memory changes from baseline in the $>45$ years age group regardless of cognitive status; however, this association was not significant for between-group analysis [36].

Although some participants in the study were in the 18-35 years age group and therefore out of the scope of this review, it is still interesting to note that Yurko-Mauro et al [36] found that supplementation with one g/day omega-3 PUFA significantly improved episodic memory, semantic memory, and working memory, regardless of cognitive status at baseline. Further subgroup analysis showed that for episodic memory, DHA intake above the mean DHA level studied (580mg/day) resulted in significant improvements in all study participants $(\mathrm{P}<0.009)$ and in the MCI group $(\mathrm{P}<0.019)$ but not in the cognitively healthy group. Further analysis determined that the effect appeared to be driven by studies where supplementation was 501-999mg DHA / day.

A meta-analysis of prospective cohort studies [37], representing level three evidence, found that a higher dietary intake of omega-3 PUFAs was not significantly associated with a lower risk of AD when compared to a lower dietary intake ( $\mathrm{RR}=0.8,95 \% \mathrm{CI} 0.74-1.08)$. It was also determined that a higher intake of fish was associated with a 36\% (95\% CI 8-56\%) lower risk of AD. The association was stronger in studies where fish intake was at least $500 \mathrm{~g} /$ week and the follow up duration was 5 
years or more. The dose response meta-analysis observed that an increment of $100 \mathrm{~g}$ of fish intake per week was associated with a lower risk of $\mathrm{AD}(\mathrm{RR}=0.89,95 \% \mathrm{CI} 0.79-0.99)$.

A further seven systematic reviews representing level three or level four evidence were identified [2, 36, 38-42], with many studies assessed by these authors included in meta-analyses discussed above. Outcomes of these systematic reviews included insufficient evidence for an association between omega-3 PUFA and cognitive decline [42], a possible association between high consumption of omega-3 PUFA and less cognitive decline [2, 39, 40], a modest benefit in slowing cognitive decline for individuals without dementia [38], improvements in some cognitive domains for MCI but not advanced AD [41], a beneficial association between omega-3 PUFA and memory function in older adults [36], through meta-analysis that total omega-3 PUFA (ES $=-0.46$, $\mathrm{P}=.001$ ), were decreased in patients with dementia compared to healthy subjects [43].

As the results of further RCTs becoming available for the combining of studies and metaanalyses of data, there is some evidence that omega-PUFA may have an effect within specific cognitive domains, such as episodic memory for MCI participants. There is also some evidence that doses above 1g/day omega-3 PUFA may have a beneficial effect on episodic memory regardless of cognitive status [36]; however the results of the available studies showed no benefit for cognitive function with omega-3 PUFA supplementation among cognitively healthy older people. Longer studies of 12 months minimum, but preferably longer are required, with appropriate placebos, where greater measurable changes in cognitive function may occur, to enable evaluation of the effects of omega-3 PUFA in preventing cognitive decline. Further consideration to overall fat consumption across life stages should be addressed, as evidence from epidemiological studies has suggested that elevated saturated fatty acids could have negative effects on age-related cognitive decline and MCI and a reduced risk of cognitive decline with high intake of monounsaturated fatty acids and PUFA, particularly omega-3 PUFA [30].

There continues to be an extensive ongoing series of studies assessing the role of dietary components in cognitive decline. While this has enabled the pooling of results and the critical appraisal of studies in a series of systematic reviews and meta-analyses, this has not enabled definitive conclusions. Limitations include the heterogeneity between studies as highlighted through the systematic reviews, where study duration often ranged from weeks to years, the age of participants ranging from 40 - 100 years, and large variations in supplement doses and the enormous range of cognitive tests used. Furthermore, the methodological approaches used to measure cognitive performance domains need to be more uniform and selected to ensure cognitive outcome measures are sensitive enough to detect treatment effects in the required cognitive domain. For example, Macready et al [44] examined the methodological approaches used to determine the effects of flavonoid supplementation on cognitive performance in humans and found that the 15 existing human dietary intervention studies assessed used a total of 55 different cognitive tests over a broad range of cognitive domains. The current standardized neuropsychological tests used for nutritional interventions has also been discussed as potentially being ill-suited for detecting subtle cognitive changes that may be expected from nutritional interventions [45]. Thus, consensus on an agreed clinically relevant battery of neuropsychological tests appropriate for nutritional outcomes delivered over an agreed time frame would enable comparison across studies. 


\section{CONCLUSION}

This review provides the current state of knowledge from systematic reviews on the effects on cognitive decline in older adults of acetyl-L-carnitine, alpha-lipoic acid, choline, inositol compounds, omega-3 PUFA or polyphenols, which are all commonly studied nutrients for neurocognitive effects. While the reviews on acetyl-L-carnitine, alpha-lipoic acid, choline and inositol compounds focused on cognitive decline, the studies of polyphenols and omega-3 PUFA's also included studies assessing cognitive performance. Many of the systematic reviews representing level 1 evidence assessed many of the same studies; thus, less weight can be given to the conclusions of this review. A critical evaluation of the current evidence available from systematic reviews indicated that there are no clinically-relevant effects from supplementation with these nutrients as a neuroprotective therapy for delaying the onset of cognitive decline in older adults.

List of Abbreviations: AD, Alzheimer's Disease; ADAS-cog, Alzheimer's Disease Assessment Scale-Cognitive Subscale; ALC, Acetyl-L-carnitine; ALA, Alpha lipoic acid; APOE, apolipoprotein E; CGI, Clinical Global Impression; DHA, Docosahexanoic acid; EPA, Eicosapentaenoic acid; JBI, Joanna Briggs Institute, Australia; MCI, Mild cognitive impairment; MMSE, Mini-Mental State Examination; PUFA, Polyunsaturated fatty acid; RCT, Randomised controlled trial; RR, Relative risk.

Competing Interests: The authors both contributed to the design of the review, the analysis of evidence and to the writing and editing of the final manuscript. None of the authors have a conflict of interest.

Acknowledgements and Funding: The authors gratefully acknowledge Professor Michael Fenech and Dr Lance Macaulay for their expert advice. This work was financially supported by the CSIRO Preventative Health Flagship.

\section{REFERENCES}

1. Virmani A, Pinto L, Binienda Z, Ali S: Food, nutrigenomics, and neurodegeneration-neuroprotection by what you eat! Mol Neurobiol 2013, 482:353-362.

2. Williams JW, Plassman BL, Burke J, Benjamin S: Preventing Alzheimer's disease and cognitive decline. Evidence report/technology assessment (Full report) 2010, 193:1-727.

3. Mucke L: Neuroscience: Alzheimer's disease. Nature 2009, 4617266:895-897.

4. Lopes da Silva S, Vellas B, Elemans S, Luchsinger J, Kamphuis P, Yaffe K, Sijben J, Groenendijk M, Stijnen T: Plasma nutrient status of patients with Alzheimer's disease: Systematic review and meta-analysis. Alzheimer's \& Dementia 2014, 104:485-502.

5. Krause D, Roupas P: Effect of Vitamin Intake on Cognitive Decline in Older Adults: Evaluation of the Evidence. J Nutr Health Aging 2015, 197:745-753.

6. Krause D, Roupas P: Dietary interventions as a neuroprotective therapy for the delay of the onset of cognitive decline in older adults: an umbrella review protocol. JBI Database of Systematic Reviews and Implementation Reports 2015, 132:74-83. 
7. The Joanna Briggs Institute. Joanna Briggs Institute Reviewers' Manual: Methodology for JBI Umbrella Reviews. Adelaide: The Joanna Briggs Institute, 2014.

8. NHMRC: How to use the evidence: assessment and application of scientific evidence. National Health and Medical Research Council (NHMRC), Commonwealth of Australia 2000.

9. Petruzzella V, Baggetto LG, Penin F, Cafagna F, Ruggiero FM, Cantatore P, Gadaleta $\mathrm{MN}$ : In vivo effect of acetyl-L-carnitine on succinate oxidation, adenine nucleotide pool and lipid composition of synaptic and non-synaptic mitochondria from cerebral hemispheres of senescent rats. Arch Gerontol Geriatr 1992, 142:131-144.

10. Hudson S, Tabet N: Acetyl-L-carnitine for dementia. Cochrane Database of Systematic Reviews 2003, 2:CD003158.

11. Montgomery SA, Thal LJ, Amrein R: Meta-analysis of double blind randomized controlled clinical trials of acetyl-L-carnitine versus placebo in the treatment of mild cognitive impairment and mild Alzheimer's disease. Int Clin Psychopharmacol 2003, 182:61-71.

12. Maczurek A, Hager K, Kenklies M, Sharman M, Martins R, Engel J, Carlson DA, Munch G: Lipoic acid as an anti-inflammatory and neuroprotective treatment for Alzheimer's disease. Adv Drug Deliver Rev 2008, 6013-14:1463-1470.

13. Klugman A, Sauer J, Tabet N, Howard R: Alpha lipoic acid for dementia. Cochrane Database of Systematic Reviews 2004, 1:CD004244.

14. Talesa VN: Acetylcholinesterase in Alzheimer's disease. Mech Ageing Dev 2001, 12216:1961-1969.

15. Higgins JPT, Flicker L: Lecithin for dementia and cognitive impairment. Cochrane database of systematic reviews 2003, 3:CD001015.

16. Fenili D, Brown M, Rappaport R, McLaurin J: Properties of scyllo-inositol as a therapeutic treatment of AD-like pathology. J Mol Med 2007, 856:603-611.

17. Barak Y, Levine J, Glasman A, Elizur A, Belmaker RH: Inositol treatment of Alzheimer's disease: a double blind, cross-over placebo controlled trial. ress in Neuropsychopharmacology \& Biological Psychiatry 1996, 204:729-735.

18. Rossi L, Mazzitelli S, Arciello M, Capo CR, Rotilio G: Benefits from dietary polyphenols for brain aging and Alzheimer's disease. Neurochem Res 2008, 3312:2390-2400.

19. Mishra S, Palanivelu K: The effect of curcumin (turmeric) on Alzheimer's disease: An overview. Annals of Indian Academy of Neurology 2008, 111:13-19.

20. Ho L, Yemul S, Wang J, Pasinetti GM: Grape seed polyphenolic extract as a potential novel therapeutic agent in tauopathies. J Alzheimers Dis 2009, 162:433-439.

21. Vingtdeux V, Dreses-Werringloer U, Zhao H, Davies P, Marambaud P: Therapeutic potential of resveratrol in Alzheimer's disease. BMC Neurosci 2008, 9Suppl:S6-S6.

22. Luchsinger JA, Tang M, Siddiqui M, Shea S, Mayeux R: Alcohol Intake and Risk of Dementia. J Am Geriatr Soc 2004, 524:540-546.

23. Solfrizzi V, D'Introno A, Colacicco AM, Capurso C, Del Parigi A, Baldassarre G, Scapicchio P, Scafato E, Amodio M, Capurso A, Panza F: Alcohol consumption, mild cognitive impairment, and progression to dementia. Neurology 2007, 6821:1790-1799. 
24. Rafnsson SB, Dilis V, Trichopoulou A: Antioxidant nutrients and age-related cognitive decline: a systematic review of population-based cohort studies. Eur J Nutr 2013, 526:1553-1567.

25. Lamport DJ, Dye L, Wightman JD, Lawton CL: The effects of flavonoid and other polyphenol consumption on cognitive performance: A systematic research review of human experimental and epidemiological studies. Nutrition and Aging 2012, 11:5-25.

26. Scholey A, Owen L: Effects of chocolate on cognitive function and mood: a systematic review. Nutrition Reviews 2013, 7110:665-681.

27. Riediger ND, Othman RA, Suh M, Moghadasian MH: A Systemic Review of the Roles of n-3 Fatty Acids in Health and Disease. J Am Diet Assoc 2009, 1094:668-679.

28. Schaefer EJ, Bongard V, Beiser AS, Lamon-Fava S, Robins SJ, Au R, Tucker KL, Kyle DJ, Wilson PW, Wolf PA: Plasma phosphatidylcholine docosahexaenoic acid content and risk of dementia and Alzheimer disease: the Framingham Heart Study. Arch Neurol 2006, 6311:1545-1550.

29. Kidd PM: Omega-3 DHA and EPA for cognition, behavior, and mood: Clinical findings and structural-functional synergies with cell membrane phospholipids. Altern Med Rev 2007, 123:207-227.

30. Solfrizzi V, Frisardi V, Capurso C, D'Introno A, Colacicco AM, Vendemiale G, Capurso A, Panza F: Dietary fatty acids in dementia and predementia syndromes: epidemiological evidence and possible underlying mechanisms. Ageing Res Rev 2010, 92:184-199.

31. Sydenham E, Dangour AD, Lim WS: Omega 3 fatty acid for the prevention of cognitive decline and dementia. Cochrane Database of Systematic Reviews 2012, 6:CD005379.

32. Lim WS, Gammack JK, Van Niekerk J, Dangour AD: Omega 3 fatty acid for the prevention of dementia. Cochrane Database of Systematic Reviews 2006, 1:CD005379.

33. Lehert P, Villaseca P, Hogervorst E, Maki PM, Henderson VW: Individually modifiable risk factors to ameliorate cognitive aging: a systematic review and meta-analysis. Climacteric 2015, 185:678-689.

34. Mazereeuw G, Lanctot KL, Chau SA, Swardfager W, Herrmann N: Effects of omega-3 fatty acids on cognitive performance: a meta-analysis. Neurobiol Aging 2012, 337:1482.e1417-1482.e1429.

35. Jiao J, Li Q, Chu J, Zeng W, Yang M, Zhu S: Effect of n-3 PUFA supplementation on cognitive function throughout the life span from infancy to old age: a systematic review and meta-analysis of randomized controlled trials. Am J Clin Nutr 2014, 1006:1422-1436.

36. Yurko-Mauro K, Alexander DD, Van Elswyk ME: Docosahexaenoic Acid and Adult Memory: A Systematic Review and Meta-Analysis. PLoS One 2015, 103.

37. Wu S, Ding Y, Wu F, Li R, Hou J, Mao P: Omega-3 fatty acids intake and risks of dementia and Alzheimer's disease: A meta-analysis. Neurosci Biobehav Rev 2015, 48:19.

38. Fotuhi M, Mohassel P, Yaffe K: Fish consumption, long-chain omega-3 fatty acids and risk of cognitive decline or Alzheimer disease: a complex association. Nature Clinical Practice Neurology 2009, 53:140-152.

39. Issa AM, Mojica WA, Morton SC, Traina S, Newberry SJ, Hilton LG, Garland RH, MacLean $\mathrm{CH}$ : The efficacy of omega-3 fatty acids on cognitive function in aging and dementia: A systematic review. Dement Geriatr Cogn Disord 2006, 212:88-96. 
40. Maclean CH, Issa AM, Newberry SJ, Mojica WA, Morton SC, Garland RH, Hilton LG, Traina SB, Shekelle PG: Effects of omega-3 fatty acids on cognitive function with aging, dementia, and neurological diseases. Evidence report/technology assessment (Summary) 2005, 114:1-3.

41. de Souza Fernandes DP, Canaan Rezende FA, Rocha GP, Filgueiras MDS, Silva Moreira PR, Goncalves Alfenas RdC: Effect of eicosapentaenoic acid and docosahexaenoic acid supplementations to control cognitive decline in dementia and Alzheimer's disease: a systematic review. Nutr Hosp 2015, 322:528-533.

42. Dangour AD, Whitehouse PJ, Rafferty K, Mitchell SA, Smith L, Hawkesworth S, Vellas B: B-Vitamins and Fatty Acids in the Prevention and Treatment of Alzheimer's Disease and Dementia: A Systematic Review. Journal of Alzheimers Disease 2010, 221:205-224.

43. Lin PY, Chiu CC, Huang SY, Su KP: A Meta-Analytic Review of Polyunsaturated Fatty Acid Compositions in Dementia. J Clin Psychiatry 2012, 739:1245-1254.

44. Macready AL, Kennedy OB, Ellis JA, Williams CM, Spencer JP, Butler LT: Flavonoids and cognitive function: a review of human randomized controlled trial studies and recommendations for future studies. Genes Nutr 2009, 44:227-242.

45. Monti JM, Moulton CJ, Cohen NJ: The role of nutrition on cognition and brain health in ageing: a targeted approach. Nutr Res Rev 2015, 282:167-180. 